hep-th/9603009, TAUP-2324-96

\title{
Interactions of strings and D-branes from M theory
}

\author{
Ofer Aharonyl], Jacob Sonnenschein ${ }^{1}$ and Shimon Yankielowicz ${ }^{1}$ \\ School of Physics and Astronomy \\ Beverly and Raymond Sackler Faculty of Exact Sciences \\ Tel-Aviv University \\ Ramat-Aviv, Tel-Aviv 69978, Israel
}

We discuss the relation between M theory and type II string theories. We show that, assuming "natural" interactions between membranes and fivebranes in M theory, the known interactions between strings and D-branes in type II string theories arise in appropriate limits. Our discussion of the interactions is purely at the classical level. We remark on issues associated with the $\mathrm{M}$ theory approach to enhanced gauge symmetries, which deserve further investigation.

$3 / 96$

1 Work supported in part by the US-Israel Binational Science Foundation, by GIF - the German-Israeli Foundation for Scientific Research, and by the Israel Academy of Science.

2 Work supported in part by the Clore Scholars Programme. Address after Sept. 1, 1996 : Department of Physics and Astronomy, Rutgers University, Piscataway, NJ 08855-0849. E-mail address : oferah@post.tau.ac.il. 


\section{Introduction}

The idea that string theory should be reformulated in terms of an eleven dimensional theory, whose low energy limit is eleven dimensional supergravity, has been revived in the past year in the form of $\mathrm{M}$ theory. Even though the fundamental formulation of this theory is still not known, the assumption of its existence has led to a simpler understanding of many types of string dualities whose origin is otherwise obscure. It is thus tempting to believe that a consistent quantum description of $\mathrm{M}$ theory does indeed exist. This is also suggested by the possible "definition" of $\mathrm{M}$ theory as the strong coupling limit of the type IIA string theory.

In this paper we discuss the relationship between $\mathrm{M}$ theory and type II string theories. We will try to learn from string theory more about the fundamental formulation of $\mathrm{M}$ theory. The original motivation for an eleven dimensional origin for string theory came from the existence of a supergravity theory in eleven dimensions, which reduces to the type IIA supergravity theory upon dimensional reduction to ten dimensions. This suggests that the low energy effective description of $M$ theory should be eleven dimensional supergravity, but says nothing about its fundamental objects. The major advance in the past year came from the realization that the BPS saturated $p$-branes of string theory, both perturbative and solitonic, may all be described by assuming the existence of a membrane and a fivebrane in $\mathrm{M}$ theory [1,2,3]. The membrane couples to the 3-form field of eleven dimensional supergravity, while the fivebrane couples to its dual. The low-energy effective actions for the extended BPS saturated $p$-branes in string theory were also connected to $\mathrm{M}$ theory [4, [0, by assuming that the action of the membrane is just the supermembrane action [6], and that the fivebrane is the solitonic fivebrane of eleven dimensional supergravity. All of these relations were derived at the level of the effective action. For this it does not matter whether the membrane, for instance, is fundamental (as in supermembrane theory), or whether it is a solitonic $p$-brane of some deeper fundamental theory. The first possibility is supported by the fact that the solitonic solution of eleven dimensional supergravity corresponding to the membrane is singular.

Our goal in this paper is to take the relation between $\mathrm{M}$ theory and string theories beyond the level of the spectrum, and discuss the interactions in the two theories. We will assume that the interactions of membranes in $\mathrm{M}$ theory are correctly described by supermembrane theory, and that the fivebranes in M theory may be described as D-branes on which membranes may have boundaries [7:4, 8]. Our discussion of RR $p$-branes in string 
theory will be based on their formulation by Polchinski [9] as boundaries for open strings (see [10] for a recent review). Of course, since we have no quantum formulation of supermembrane theory, our discussion throughout this paper will be purely classical. Since interactions necessarily involve non-BPS saturated states, quantum corrections could be important. However, at the classical level we will be able to show, using known results, that by assuming the above natural interactions in $\mathrm{M}$ theory we get the correct interactions between strings and $p$-branes (for $p \leq 6$ ) when we go over to the string theory limit. We believe that this presents stronger evidence for the existence of a quantum $\mathrm{M}$ theory, whose classical limit gives supermembrane theory, at least as an effective action. This could be some version of supermembrane theory which avoids the problem of the continuous spectrum (such as a membrane with thickness [1, 11]), a string theory for which the membrane action is a low-energy effective description [12], or a completely different theory which we have not yet been able to imagine.

In section 2 we describe the known relations between $M$ theory and type IIA string theory, and show how they lead to the same interactions in the two theories. In section 3 we perform the same analysis for the type IIB string theory in ten dimensions. In section 4 we discuss some issues, associated with the description of D-branes in string theory, whose M theory interpretation is still unclear. Understanding these issues may help toward the formulation of $\mathrm{M}$ theory. We end in section 5 with a summary and some open questions.

\section{Interactions of type IIA string theory from M theory}

The simplest relation between $M$ theory and a string theory arises when we compactify one dimension, which we will denote by $X^{11}$, on a circle of radius $R_{11}$. In the limit of small $R_{11}$, this is believed to lead to the ten-dimensional type IIA string theory, with a string coupling $e^{\phi_{A}}$ proportional to $R_{11}^{3 / 2}$ [1,2] (we will ignore numerical constants throughout this paper, as well as the tensions of the string and of the membrane which set the scale in relations of this type). We will begin this section by reviewing the evidence for this relationship, and continue by generalizing it also to the interactions of the type IIA string with D-branes.

The simplest evidence for the relation between M theory and type IIA strings comes from the low-energy effective action. It is just the statement that the dimensional reduction 
of 11 dimensional supergravity gives 10 dimensional type IIA supergravity, when we identify the 11 dimensional metric with the 10 dimensional metric, RR 1-form and dilaton by

$$
g_{m n}^{(11)} d x^{m} d x^{n}=e^{-2 \phi_{A} / 3} g_{\mu \nu}^{(10)} d x^{\mu} d x^{\nu}+e^{4 \phi_{A} / 3}\left(d x^{11}-A_{\mu} d x^{\mu}\right)^{2}
$$

(throughout this paper we will use $m, n, \cdots$ for 11 dimensional indices, $\mu, \nu, \cdots$ for 10 dimensional indices, and $\alpha, \beta, \cdots$ for 3 dimensional indices on the worldvolume of the membrane). We assume, as usual, that none of the background fields depend on $X^{11}$. The 2 -form $B_{\mu \nu}$ and 3-form $A_{\mu \nu \lambda}$ of type IIA string theory are related to the 3 -form $A_{m n k}^{M}$ of 11 dimensional supergravity simply by $A_{\mu \nu \lambda}=A_{\mu \nu \lambda}^{M}$ and $B_{\mu \nu}=A_{\mu \nu(11)}^{M}$. For this relation between the theories we need only to use the fact that the low-energy effective description of $\mathrm{M}$ theory is given by 11 dimensional supergravity.

Additional evidence for the relation between $M$ theory and type IIA string theory comes from the identification [1,2, 3] of the BPS saturated $p$-branes of the two theories, for $p \leq 63$, and of the effective actions describing these $p$-branes. For this we need to assume a particular spectrum of $p$-branes in the 11 dimensional $\mathrm{M}$ theory, as well as the effective actions describing them. It turns out that the identification goes through if we use the membrane and fivebrane $\theta_{\text {of }}$ supermembrane theory [6] as the only BPS saturated $p$-branes of $\mathrm{M}$ theory.

First, it has long been known [14] that the double dimensional reduction of the supermembrane worldvolume theory gives the type IIA superstring worldsheet theory, at least on the classical level (our discussion throughout this paper will be purely classical, since we do not know how to quantize supermembrane theory). Thus, a supermembrane for which one worldvolume coordinate is always wrapped around $X^{11}$ is, at least classically and in the $R_{11} \rightarrow 0$ limit, exactly the same as a type IIA superstring.

A more complicated identification relates a supermembrane which is not wrapped around $X^{11}$ with the D-2-brane of type IIA string theory. The two effective actions in this case turn out to be the same [4.5] if we perform a three dimensional duality transformation

3 The issue of $p$-branes with $p>6$ and their relation to $\mathrm{M}$ theory is more complicated [3, 13, and will not be discussed here.

4 To avoid unnecessary confusion, we will use the word "fivebrane" to describe the 5-brane of M theory, and the word "5-brane" to describe the 5-branes of string theory. The word "membrane" or "supermembrane" will denote the 2-brane of M theory, and the word "2-brane" will denote the 2-brane of string theory. 
on the worldvolume of the membrane, transforming the scalar $X^{11}$ into a vector field $A_{\alpha}$. Since the only dependence of the supermembrane action on $X^{11}$ is through its kinetic term, we can treat $w_{\alpha}=\partial_{\alpha} X^{11}$ as a fundamental field if we add to the action a Lagrange multiplier term of the form $\epsilon^{\alpha \beta \gamma} \Lambda_{\alpha} \partial_{\beta} w_{\gamma}$. By solving the equation of motion of $w_{\gamma}$ we find that $F_{\alpha \beta}=\partial_{\alpha} \Lambda_{\beta}-\partial_{\beta} \Lambda_{\alpha}$ behaves as a gauge field strength (together with $B_{\alpha \beta}$ defined below), and the action of the supermembrane becomes exactly the effective action of the Dirichlet 2-brane. This includes the Born-Infeld term $\sqrt{-\operatorname{det}\left(G_{\alpha \beta}+F_{\alpha \beta}-B_{\alpha \beta}\right)}$, where $G_{\alpha \beta}=\partial_{\alpha} x^{\mu} \partial_{\beta} x^{\nu} g_{\mu \nu}^{(10)}$ and $B_{\alpha \beta}=\partial_{\alpha} x^{\mu} \partial_{\beta} x^{\nu} B_{\mu \nu}$. The equation of motion of $w_{\alpha}$ sets

$$
w_{\alpha} \sim e^{-\phi_{A}} \epsilon_{\alpha \beta \gamma}\left(F^{\beta \gamma}-B^{\beta \gamma}\right)
$$

up to factors involving the metric on the supermembrane which we ignore. The fermionic terms in (2.2), and throughout the paper, are suppressed, but we do not expect their analysis to present any fundamental difficulties. They should just supersymmetrize the bosonic terms. Note that the holonomy of the gauge field, when the 2-brane has topologically non-trivial cycles, is not determined by this transformation. Other global issues involved in this transformation are discussed below.

The D-4-brane and NS 5-brane of type IIA string theory are similarly related to the wrapped or unwrapped "magnetic" fivebranes of $M$ theory [3,4]. In this case an exact expression for the effective worldvolume action of the fivebrane is not available, since it involves an anti-self-dual 3-form field strength. However, the fields along the D-4-brane and the NS 5-brane are correctly given by the double and single dimensional reductions of the "magnetic" fivebrane fields. The tensions of these $p$-branes, as well as the tensions of the string and membrane described in the previous paragraphs, also have the correct dependence on the string coupling constant [3], using the relations between their descriptions in $\mathrm{M}$ theory and in string theory and equation (2.1).

The other BPS saturated $p$-branes of the type IIA string theory with $p \leq 6$ are the Dirichlet 0-brane and 6-brane. These arise in $\mathrm{M}$ theory as "electric" and "magnetic" Kaluza-Klein excitations [1,2,2,3]. The 0-branes are characterized just by their mass, which is the same in both of their interpretations [2]. For the 6-branes the tensions in both interpretations are proportional (in the string metric) to $e^{-\phi_{A}}$, and it is known that the Kaluza-Klein monopole in 11 dimensional supergravity breaks half of the supersymmetries [15]. It should be possible to derive the low energy effective action of the 6 -brane from the Kaluza-Klein monopole solution to the supergravity theory, as done for the membrane and 
the fivebrane in [16]. Obviously there are 3 zero modes corresponding to transverse motion of the 6-brane, and the other 5 bosonic zero modes should correspond to the Born-Infeld vector field in the worldvolume of the 6-brane. We expect this vector field to arise from fluctuations in the 3 -form field $A_{m n k}^{M}$ around the monopole solution, in the same way as the 2-form arises in the worldvolume theory of the fivebrane [16], but this has not yet been verified as far as we know.

The next step after identifying the spectrum of the two theories should involve checking the interactions of the various $p$-branes. The basic interactions of supermembranes in supermembrane theory are geometrical splittings and joinings of the membranes, like in superstring theory. An important difference between the two theories is that in 11 dimensional supermembrane theory there is no dimensionless coupling constant, so any "interaction vertex" is necessarily of order one, and it is not clear how to define a perturbative expansion. However, once we introduce a small compactification radius, we have a small dimensionless coupling in the theory (which is just the radius in units determined by the supermembrane tension), and it is meaningful to talk about vertices of $p$-branes which wrap around this small dimension, since these become weakly coupled. Another difference between membranes and strings is that free membranes can smoothly change their topology (in space). Such topology changes will be involved in some of the interactions described below, such as the generation of a "virtual" open string on a 2-brane.

Let us start with the string and 2-brane of type IIA string theory, both of which arise from the supermembrane in 11 dimensions. A diagram for the interaction of strings (in 10 dimensions) may be transformed into a diagram of membranes in $\mathrm{M}$ theory just by replacing each point in the diagram by a circle wrapped around the eleventh dimension. Since the tensions of the string and membrane are related simply by $T_{S}=T_{M} 2 \pi R_{11}$ (when both are written in the same metric [3]), it is obvious from the Nambu-Goto form of the action that the action associated with any diagram is the same in both descriptions. The only subtle issue here is the dependence of the diagram on the string coupling (or the dilaton), since when one performs the double dimensional reduction of the supermembrane action [14], one gets the string action coupled to the $g_{\mu \nu}^{(10)}$ and $B_{\mu \nu}$ fields but not to the dilaton. This is, of course, to be expected since this reduction is purely classical, and the dilaton term is of higher degree in $\alpha^{\prime}$ than the others. From the point of view of M theory the dilaton is just a Kaluza-Klein scalar field. We expect a correct quantum treatment of this reduction to give also the dilaton term. It is, however, not clear how to do this rigorously, since the supermembrane worldvolume action is generally not renormalizable. 
The other interaction of membranes which we can discuss at weak coupling is the interaction of a string with a 2-brane in the type IIA string theory. In the string theory, this interaction is described [9] simply by allowing the string to end on a 2-brane, as in figure 1 , where we took the 2-brane to be spherical.

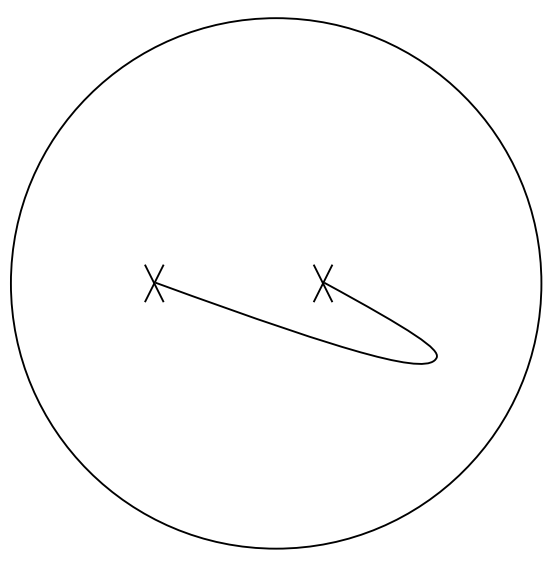

Fig. 1

The end of the string behaves (inside the 2-brane) as a particle charged with respect to the gauge field on the surface of the 2-brane. In order to get the corresponding interactions in $\mathrm{M}$ theory, we should look at diagrams of membrane interactions which include a region in which the membrane is wrapped around $X^{11}$. The string in figure 1 should be replaced by such a wrapped membrane, joined smoothly to the rest of the membrane. When we take $R_{11}$ to zero, this becomes just the interaction of the string with the 2-brane. The only thing left to check is that the end of the "string" behaves like a charged particle with respect to the gauge field living in the unwrapped part of the membrane. The end of the "stringy" part of the membrane is topologically a circle in the rest of the membrane, which is wrapped around $X^{11}$. Thus, if we perform an integral $\oint d x^{\alpha} \partial_{\alpha} X^{11}$ around the end-point of the string, it will be a non-zero constant (namely, $2 \pi R_{11}$ ). However, the identification of the 2-brane action with the membrane action (2.2) means that this is proportional to $\oint \epsilon_{\alpha \beta \gamma}\left(F^{\beta \gamma}-B^{\beta \gamma}\right) d x^{\alpha}$ which is just the electric flux emanating from the end-point of the string. Thus, we have shown that the interactions of strings and 2-branes in type IIA string theory are correctly described by $\mathrm{M}$ theory. In both cases the effective action describing the interaction includes a part corresponding to the separate actions of the string and the membrane (which are identified as above), and a contribution from the boundary of the string, or the interaction region between the membranes, which (in the $R_{11} \rightarrow 0$ limit) is just the action for the motion of a charged particle. 
Next, we should add the fivebranes of $M$ theory. These play the same role in $M$ theory as the D-branes in string theory [7, 4, 8]. The membranes can have a boundary along a fivebrane, and it behaves (in the $5+1$ worldvolume theory of the fivebrane) as a string which is charged with respect to the 2-form field living on the fivebrane (this is a limit of the dyonic string of [17]). From here we can easily see the interactions of the type IIA string with 4-branes and 5-branes. The interaction of the type IIA string with a 4-brane is just the double dimensional reduction of the interaction of a membrane with a fivebrane [4], with both worldvolumes wrapped around $X^{11}$. The boundary of the membrane becomes the boundary of a string, and it is charged with respect to the gauge field of the 4-brane (which is the dimensional reduction of the 2-form field on the fivebrane), as desired. We find no similar interactions between the string and the 5-brane, since the end of a "stringy" membrane is a circle wrapped around $X^{11}$, and there are no such cycles in the unwrapped fivebrane. This is consistent with the absence of such interactions between the string and the NS 5-brane in the type IIA string theory. There will be more complicated interactions between the string and the 5-brane when the radius of the eleventh dimension is non-zero, which will lead to scattering of strings and 5-branes, and it would be interesting to compare these with the corresponding interactions in string theory. To compute these we require a conformal field theory description of the NS 5-brane, perhaps along the lines of [18].

The other interactions which have a simple description in the type IIA string theory are between the string and the 0-brane and 6-brane. The 0-brane can be viewed simply as a collapsed membrane [4, [8], ensuring that its interactions are also the same in string theory and $\mathrm{M}$ theory. In fact, since an open string with both ends on a 0-brane is just a closed string, there are actually no purely bosonic interactions between strings and 0-branes, at least at the level of the low-energy effective action. This is clear both in the string picture, since there are no new string sectors, and in the membrane picture, since the 0-brane may be viewed as a membrane whose size has gone to zero. The Kaluza-Klein "magnetic" 6-brane of $\mathrm{M}$ theory on a circle may be viewed as a non-trivial embedding of the $S^{1}$ of the eleventh dimension in the other dimensions. In this embedding, the radius of the eleventh dimension goes to zero at the position of the 6-brane (this is also clear from the type IIA picture, where the dilaton $e^{\phi}$ vanishes at the position of the 6 -brane). Thus, a membrane wrapped around the eleventh dimension may end on the 6-brane, and in the string theory limit this would give a string ending on a Dirichlet 6-brane. An analysis of the effective field theory on the 6-brane should lead to the endpoint of this string being charged with respect to the electric field on the 6 -brane. In the 11 dimensional supergravity this electric 
field arises from zero modes of the monopole solution corresponding to turning on the 3-form field, under which the membrane is charged. Thus, this seems reasonable, but we have not checked it rigorously. Other interactions (such as interactions of 2-branes with themselves) cannot be seen at weak coupling in string theory, hence we cannot compare them. We expect that $M$ theory will also have interactions between fivebranes, but we do not know how to describe these in $\mathrm{M}$ theory or in string theory, so obviously we cannot compare them.

\section{Interactions of type IIB string theory from $M$ theory}

The other string theory which is simply related to $M$ theory is the type IIB theory (for other theories we need to put $\mathrm{M}$ theory on an orbifold, and it is not clear how the twisted sectors should be defined in M theory, though there is by now a certain amount of experience with orbifolds [19,20] which should hint at the appropriate definition). To reach type IIB string theory, we compactify two of the dimensions of $M$ theory on a torus. By the analysis of the previous section, this is just type IIA string theory on a circle, which is known to be related by $\mathrm{T}$ duality to type IIB string theory on a circle [21]. By performing this duality, we find [3] that $\mathrm{M}$ theory on a torus of area $A_{T}$ and of modular parameter $\tau$ seems equivalent to type IIB string theory on a circle of radius $R_{B} \sim A_{T}^{-3 / 4}$, with a complex coupling constant $\lambda=\chi+i e^{-\phi_{B}}$ (where $\chi$ is the $\mathrm{RR} 0$-form or axion) which equals $\tau$. Note that here we do not need to take the compact dimensions to zero in order to get a weakly coupled string theory, since the string coupling depends only on the modular parameter of the torus. We should, however, take the size of the torus to zero (with a constant modular parameter) in order to get the type IIB string theory in ten dimensions (since then $R_{B} \rightarrow \infty$ ), and this is the case we will analyze here. This means that in the context of M theory, the "stringy" description is in fact exact for type IIB theory in ten dimensions, while for type IIA it is only a small coupling (small radius) approximation. The $S L(2, \mathbf{Z})$ duality of the type IIB string theory obtains through this construction a geometrical interpretation, as the group of modular transformations of the torus on which $\mathrm{M}$ theory was compactified [22, 3].

The identification between $M$ theory on a torus and type IIB theory is based on the same type of evidence as described above for the type IIA theory. In fact, since the relation of type IIA and type IIB theories on a circle via T duality is well understood (also for the $p$ branes of the two theories), the identification of the type IIA theory is apparently sufficient 
in order to identify the type IIB theory as well. However, the weak coupling limits of the type IIB theory (in which we understand it as a string theory) are not necessarily related to weak coupling limits of the type IIA theory. Thus, more checks are actually necessary, since our identification of the interactions above was just for weak coupling. In any case, some interactions look different in the type IIB theory, so it is worthwhile to repeat the whole analysis above on the description of the $p$-branes and of their interactions.

The simplest $p$-branes in the type IIB theory are the bound states of strings and Dstrings [23]. For every pair of coprime integers $(n, m)$ there exists a BPS saturated bound state of $n$ fundamental strings and $m$ D-strings, whose worldsheet low-energy effective action is essentially that of the type IIB string. In the $\mathrm{M}$ theory, these are [3] identified with membranes with the topology of a torus, one of whose cycles is wrapped $n$ times around one cycle of the space-time torus and $m$ times around the other cycles. As described in the previous section, this gives a string with the type IIA string action in 9 dimensions, which after the $\mathrm{T}$ duality becomes the type IIB string action as desired. Membranes which are not wrapped around any cycle of the torus become wrapped around the circle of type IIB theory after the $\mathrm{T}$ duality transformation, so that they are not visible in ten dimensional type IIB theory (we will limit ourselves here only to a discussion of the states which remain in the limit of $\left.R_{B} \rightarrow \infty\right)$. Membranes which are completely wrapped around the torus are identified [3] with Kaluza-Klein particles of type IIB theory on a circle. In the tendimensional limit these become part of the ten dimensional fields. Thus, the $(n, m)$ strings are all that remain of the membrane after compactifying the space-time on a very small torus. The fivebrane, on the other hand, must be wrapped around both cycles of the torus to give a state of ten dimensional type IIB theory (it must of course have an appropriate topology for this). The difference between the two arises from the different properties of NS and R branes under T duality, as can be seen from their type IIA description. The fivebrane wrapped around the torus gives an $S L(2, Z)$ invariant 3-brane in the type IIB theory, which should be identified with the D-3-brane of this theory. The effective action of the D-3-brane is the supersymmetric generalization of the Born-Infeld action in $3+1$ dimensions. Its low-energy limit is just $N=4 U(1)$ gauge theory in $3+1$ dimensions [24,25, which is known to be $S L(2, \mathbf{Z})$ invariant. As far as we know, this action has not yet been derived from the double dimensional reduction on a torus of the fivebrane

5 When $n$ and $m$ are not coprime, these states are only marginally stable [3] and are not expected to exist as bound states. 
of M theory, but essentially this should be just the supersymmetric generalization of the dimensional reduction of [26]. Using this reduction, the integrals of the 2 -form field of the fivebrane around the two cycles of the torus give the electric and magnetic fields on the worldvolume of the 3-brane (recall that the field strength of the 2-form is anti-self-dual, so that the two integrals do not give independent 1-forms). If the fivebrane is wrapped $n$ times around the torus, the low-energy effective description should be given by the $N=4$ $U(n)$ gauge theory [23,25], which is also believed to be $S L(2, \mathbf{Z})$ invariant.

The other BPS saturated $p$-branes in the type IIB theory (with $p \leq 6$ ) are the 5branes, which are also labeled by two coprime integers $n$ and $m$. These arise in M theory as Kaluza-Klein "magnetic" branes [3], like the 6-brane in the type IIA theory.

Next, let us move on to the interactions, beginning with the interactions between a string and a 3-brane in the type IIB theory. These arise from membrane-fivebrane interactions in $\mathrm{M}$ theory, like the string-4-brane interactions described in the previous section. In this case any $(n, m)$ string can end on the 3 -brane (which is a fivebrane wrapped around the torus), since the end-cycle of the appropriate "stringy" membrane is wrapped around the $(n, m)$ cycle of the torus, and all such cycles exist in the fivebrane when it is wrapped around the torus. The end-point of such a string describes (in the 4 dimensional effective theory of the 3-brane) a dyon with electric charge $n$ and magnetic charge $m$ [25]. This arises from the coupling of the boundary of the membrane to the 2 -form of the fivebrane, as in the previous section.

The interactions of strings with 5-branes are exactly analogous to the interactions of strings with 6-branes described in the previous section. At the Kaluza-Klein 5-brane, the appropriate $(n, m)$ cycle of the torus goes to zero size (in the 11 dimensional metric), so that a membrane wrapped around this cycle may end on the 5-brane. In the string theory limit this gives an $(n, m)$ string ending on an $(n, m) 5$-brane.

The interactions of strings of a particular type $(n, m)$, when the coupling is such that these strings are weakly coupled, arise from $\mathrm{M}$ theory in the same way as the interactions of type IIA strings described above. More interesting is the interaction between strings of different types, for instance between a $(1,0)$ fundamental string and a $(0,1)$ D-string when the fundamental string is weakly coupled (the generalization to general strings is straightforward). Let us look at a generic interaction in the type IIB string theory, such as the scattering of a string on a D-string [27], described (in spacetime) in figure 2. 


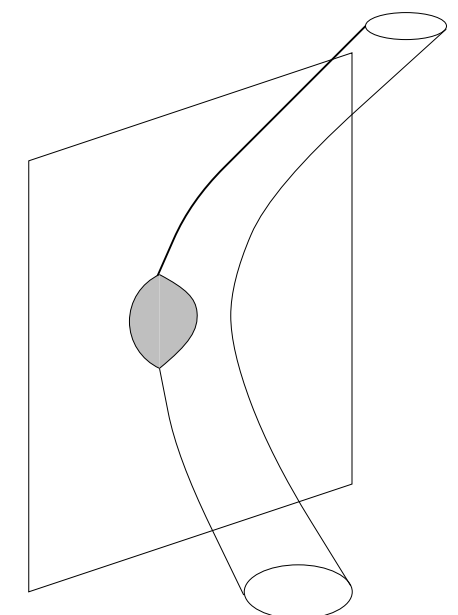

Fig. 2

When a fundamental string has end-points on a D-string, these are charges on the worldsheet of the D-string, so that there is a constant electric field between the two endpoints. Thus, according to the description of D-strings in [23], the portion of the D-string between the two end-points is actually a $(1,-1)$ string, and the time slices of the interaction actually look as in figure 3a (taking the D-string also to be a finite closed string). How can we understand such an interaction from M theory ? In the beginning we have two toroidal membranes, one with a cycle wrapped around the $(1,0)$ cycle of the spacetime torus and another with a cycle wrapped around the $(0,1)$ cycle of the spacetime torus. Then, they join together to give a genus 2 surface, as in figure $3 \mathrm{~b}$.

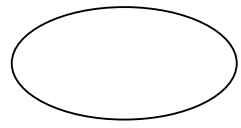

$(0,1)$

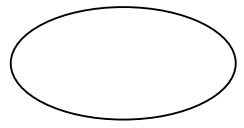

$(1,0)$

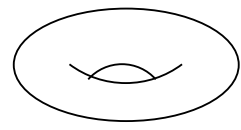

$(0,1)$

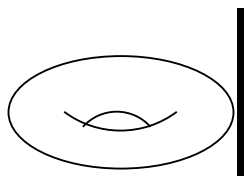

$(1,0)$
$(0,1)$

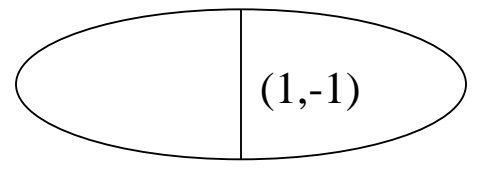

$(1,0)$

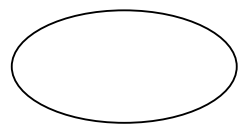

$(0,1)$

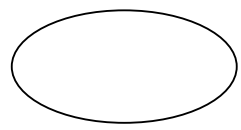

$(1,0)$

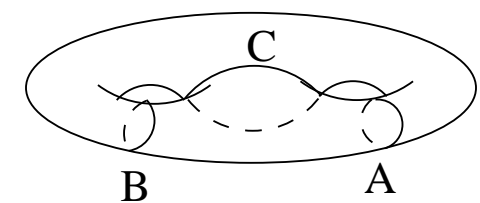

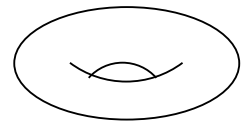

$(0,1)$

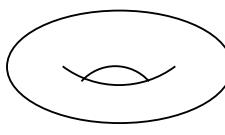

$(1,0)$

Fig. 3a

When we take the spacetime torus to zero, the stringy description of this interaction becomes exactly that of figure 3a. The "middle" cycle (denoted by $\mathrm{C}$ in figure $3 \mathrm{~b}$ ) of 
the genus 2 surface is homologically the difference between the cycles A and B, and thus is wrapped around the $(1,-1)$ cycle of the spacetime torus, as desired. Thus, the basic interactions of strings and D-strings are also correctly described by the basic interaction between membranes in M theory.

\section{Remarks on enhanced gauge symmetries in $M$ theory}

The description of D-strings in M theory, as in the previous section, seems very different from their description in [23]. Witten described the effective theory of the $(n, m)$ string as a $U(n)$ gauge theory with $m$ quarks at infinity. However, since for coprime $n$ and $m$ this theory is believed to have a mass gap, the low-energy degrees of freedom (which are the only ones we are discussing here) are only those in the $U(1)$ gauge superfield (including the scalars corresponding to the position of the D-string), which we get also from the M theory description. As we discussed in the previous section, we can replace one of the compact dimensions of the torus by a gauge field on the worldvolume of the membrane. When we go over to the string picture, this becomes a gauge field on the worldsheet of the D-string, which has (in the "stringy" limit) a constant value corresponding to the winding number around the appropriate compact dimension. Thus, we get exactly the same description as in [23].

However, we should also be able to discuss configurations of $n(1,0)$ D-strings (for instance). According to [23], such configurations are described by a $U(n)$ gauge theory on the worldsheet of the D-string. How does this arise in M theory ? In string theory, when we have two parallel D-strings, we get a W-boson state from an open fundamental string connecting the two. In $\mathrm{M}$ theory, therefore, we expect a "wormhole" configuration between the two membranes to give rise to the enhanced gauge symmetry. Whenever two membranes touch each other, the worldvolume scalars describing the position of the two membranes should become a $2 \times 2$ matrix [23]. Perhaps this can be understood by examining the possible interactions of the two membrane sheets at this point. Even though in string theory there is no geometrical interpretation of this phenomenon, we believe that such an interpretation may be possible in $\mathrm{M}$ theory, but we have not been able to find it. Another possibility is that the enhanced gauge symmetry may only be understood in $\mathrm{M}$ theory at the quantum level. This is reasonable, since the additional states appear in the effective action only when the distance between the D-branes is much smaller than the appropriate "Planck length". Since we do not know how to quantize the M theory, 
it is difficult to explore this possibility. Note that the same mechanism should give the enhanced gauge symmetry for the D-strings in the type IIB theory and for the D-2-branes of the type IIA theory discussed in the previous section. For fivebranes in M theory the enhanced gauge symmetry arises [7] from tensionless strings [28] which appear when the distance between the fivebranes goes to zero. The winding states of these strings become additional gauge bosons. In string theory this leads to the enhanced gauge symmetries which occur for the D-4-branes of the type IIA theory and for the D-3-branes of the type IIB theory. Perhaps we may be able to understand the analogous problem for membranes in a similar way, since the boundary of one membrane on another membrane is also a string, whose tension goes to zero when two membranes approach each other.

The enhanced symmetry described above should arise only for two membranes which have the same orientation. Membranes with different orientations go over, in the string theory, to a D-brane anti-D-brane pair, and then we expect a tachyonic instability to develop when they approach each other [29]. Obviously, the geometry of the connecting "wormholes" is different in the two cases. Hence, it is possible that such an instability may indeed exist for oppositely oriented membranes, but we do not know how to derive it from $M$ theory. Perhaps it can only be seen in the quantum theory which we do not yet know how to formulate.

At this point we should comment that if the $S L(2, \mathbf{Z})$ symmetry of type IIB string theory is exact (this is obvious if the type IIB string is indeed exactly described by $M$ theory on a torus), there should be no difference between the fundamental string and the D-string. Any apparent difference between the two must come from the fact that one of them is (in the usual description) weakly coupled, while the other is strongly coupled. Differences which do not depend on the string coupling, for instance in the spectrum of BPS saturated states, cannot exist. At weak coupling it seems that the string world-sheet action is fundamental, while the D-string worldsheet action arises from open strings coupled to the D-string. A reciprocal picture should give the correct description at strong coupling. As described in the previous section, the interactions of the strings and of the D-strings seem to be the same. Thus, the gauge field which appears in the effective theory of the D-string and not in the fundamental theory of the string does not play any dynamical role. One of the apparent differences between strings and D-strings is that for $n$ D-strings which sit on top of each other we get an enhanced $U(n)$ gauge group, while no similar phenomenon is known for fundamental strings. This leads, for instance, to the fact 10 that there is 
no distinction between a D-string wrapped twice around a space-time circle or two Dstrings wrapped once around the same circle. For fundamental strings the corresponding perturbative states are clearly distinct. So far we have not understood how to get this enhanced gauge group from M theory, but it seems that if it arises for D-strings it should arise also for fundamental strings, since the description of the two in $M$ theory should be the same. It does not seem reasonable, though we have not been able to rigorously rule out this possibility, that this gauge symmetry would only arise for strongly coupled strings. Of course, in strong coupling the additional states giving rise to the enhanced gauge symmetry may be described by open D-strings connecting the fundamental strings. This is just the $S L(2, \mathbf{Z})$ dual of the picture described in 23.

\section{Summary and future directions}

In this paper we examined the correspondence between the interactions in M theory and in type II string theories. We found that the basic interactions between membranes and membranes and between membranes and fivebranes in $\mathrm{M}$ theory give rise to the known interactions of strings and D-p-branes (with $p \leq 6$ ) in type II string theory, in appropriate weak coupling limits. The understanding of $p$-branes with $p>6$ in $\mathrm{M}$ theory is still not clear. In ten dimensions $p$-branes with $p>6$ affect the asymptotic form of the metric (at least when they are flat), so that they cannot appear in flat ten dimensional spacetime. Thus, to discuss them we need to study $M$ theory on curved space. Another possible generalization of our work is to orbifolds of $\mathrm{M}$ theory [19,20], which are believed to describe heterotic strings. The analysis of the bulk interactions in these theories is similar to the analysis we performed here, but new issues arise at the orbifold fixed points, which are beyond the scope of this paper. Type I strings also appear in some of these theories, which are not BPS saturated states [30, 19]. It is not clear how to analyze the interactions of these strings in M theory.

Our analysis was limited, by the level of our knowledge of $\mathrm{M}$ theory, to a classical analysis of low-energy effective actions. Such an analysis should certainly not be enough to describe compactifications with radii much smaller than the 11 dimensional Planck length, as we have attempted to do here. It is not clear if anything can be done beyond this level without knowing a quantum formulation for $\mathrm{M}$ theory. Perhaps a systematic analysis of the membrane corrections to type IIA string theory at non-zero coupling, for instance, may be possible just by assuming that $\mathrm{M}$ theory is supermembrane theory. Even 
at the low-energy level, the description of some string theory effects, such as enhanced $U(n)$ gauge symmetry, in M theory is still not clear. These effects may arise only at the quantum level, but they may also correspond to classical effects which have not yet been investigated. In any case, we hope that our work will lead to a better understanding of $\mathrm{M}$ theory and of the correct way to formulate it.

We end with an amusing numerical observation about $p$-branes in $\mathrm{M}$ theory. In our discussion we used the fact that membranes in M theory can have boundaries on fivebranes, or on other membranes. Let us raise the question whether membranes can have boundaries on any other, so far unknown, $p$-branes in M theory. The boundary of a membrane is a string, so it should couple to a 2 -form $B_{\alpha \beta}$ in the worldvolume theory of the $p$-brane. A BPS saturated $p$-brane breaks half the supersymmetries. In 11 (or 10) dimensions this means it should have 16 normalizable fermionic zero modes, and correspondingly (by supersymmetry) 8 normalizable bosonic zero modes. These zero modes correspond to the bosonic physical degrees of freedom on the worldvolume of the $p$-brane. If we assume that the only degrees of freedom on the $p$-brane in 11 dimensions are the 2 -form and the transverse excitations (corresponding to the motion of the $p$-brane in 11 dimensions), we find the equation

$$
\frac{((p+1)-2)((p+1)-3)}{2}+(11-(p+1))=8
$$

whose solutions give $p=2$ or $p=3$. If $p=5$ there is an additional possibility of having a self-dual or anti-self-dual 2-form. This halves the number of degrees of freedom in the 2 -form and leads to the additional solution $p=5$. Allowing additional worldvolume gauge fields does not add any new solutions. The solutions $p=2,5$ correspond to the known $p$-branes of $\mathrm{M}$ theory, but the interpretation of the $p=3$ solution is not clear. Of course, such "hand-waving" arguments do not prove that such 3-branes indeed exist (there are no corresponding solitons of 11 dimensional supergravity as far as we know). For instance, using a similar computation for strings, whose ends couple to 1-form fields, we would find that Dirichlet $p$-branes with any value of $p$ are possible. However, only half of the Dirichlet $p$-branes of type II theories, and less in type I theory, can consistently couple to superstrings. If such a 3-brane exists, it would look like a 12 dimensional object, since a 2form field in $3+1$ dimensions is dual to a scalar. As in our analysis of strings and 2-branes in section 2, the membrane could then be interpreted as a 3-brane wrapped around the twelfth dimension, and the interaction of the membrane with the 3-brane would be simply 
a Nambu-Goto type interaction for the 3-brane. The relation of this observation to recent discussions of 12 dimensional theories [31, 12,24] is still not clear.

\section{Acknowledgments}

We thank O. Ganor, Y. Oz, J. Pawelczyk and P. K. Townsend for discussions, and M. B. Green for comments and for reading this manuscript. 


\section{References}

[1] P. K. Townsend, "The eleven dimensional supermembrane revisited", Phys. Lett. 350B (1995) 184, hep-th/9501068

[2] E. Witten, "String theory dynamics in various dimensions", Nucl. Phys. B443 (1995) 85, hep-th/9503124

[3] J. H. Schwarz, "An $S L(2, \mathbf{Z})$ multiplet of type II superstrings", Phys. Lett. 360B (1995) 13, hep-th/9508143; "Superstring dualities", hep-th/9509148; "The power of M theory", Phys. Lett. 367B (1996) 97, hep-th/9510086; "M theory extensions of T duality", hep-th/9601077

[4] P. K. Townsend, "D-branes from M-branes", hep-th/9512062

[5] C. Schmidhuber, "D-brane actions", hep-th/9601003

[6] E. Bergshoeff, E. Sezgin and P. K. Townsend, "Properties of the eleven dimensional supermembrane theory", Phys. Lett. 189B (1987) 75

[7] A. Strominger, "Open $p$-branes", hep-th/9512059

[8] K. Becker and M. Becker, "Boundaries in M theory", hep-th/9602071

[9] J. Polchinski, "Dirichlet branes and Ramond-Ramond charges", Phys. Rev. Lett. 75 (1995) 4724, hep-th/9510017

[10] J. Polchinski, S. Chaudhuri and C. V. Johnson, "Notes on D-branes", hep-th/9602052

[11] F. Aldabe and A. L. Larsen, "Supermembranes and superstrings with extrinsic curvature", hep-th/9602112

[12] D. Kutasov and E. Martinec, "New principles for string/membrane unification", hepth/9602049

[13] E. Bergshoeff, M. De Roo, M. B. Green, G. Papadopoulos and P. K. Townsend, "Duality of type II 7-branes and 8-branes", hep-th/9601150

[14] M. J. Duff, P. S. Howe, T. Inami and K. S. Stelle, "Superstrings in $D=10$ from supermembranes in $D=11$ ", Phys. Lett. 191B (1987) 70

[15] S. K. Han and I. G. Koh, " $N=4$ remaining supersymmetry in Kaluza-Klein monopole background in $D=11$ supergravity theory", Phys. Rev. D31 (2503) 1985

[16] D. M. Kaplan and J, Michelson, "Zero modes for the $D=11$ membrane and fivebrane", Phys. Rev. D53 (3474) 1996, hep-th/9510053

[17] M. J. Duff, S. Ferrara, R. R. Khuri and J. Rahmfeld, "Supersymmetry and dual string solitons", hep-th/9506057

[18] D. Kutasov, "Orbifolds and solitons", hep-th/9512145

[19] P. Horava and E. Witten, "Heterotic and type I string dynamics from eleven dimensions", Nucl. Phys. B460 (1996) 506, hep-th/9510209

[20] K. Dasgupta and S. Mukhi, "Orbifolds of M theory", hep-th/9512196; E. Witten, "Five-branes and M theory on an orbifold", hep-th/9512219; A. Sen, "M theory on 
$\left(K 3 \times S^{1}\right) / \mathbf{Z}_{2}$ ", hep-th/9602010; A. Kumar and K. Ray, "M-theory on orientifolds of $K 3 \times S^{1}$, hep-th/9602144

[21] J. Dai, R. Leigh and J. Polchinski, "New connections between string theories", Mod. Phys. Lett. A4 (1989) 2073

[22] P. S. Aspinwall, "Some relationships between dualities in string theory", hepth/9508154

[23] E. Witten, "Bound states of strings and p-branes", Nucl. Phys. B460 (1996) 335, hep-th/9510135

[24] A. A. Tseytlin, "Self duality of the Born-Infeld action and Dirichlet 3-branes in type IIB string theory", hep-th/9602064

[25] M. B. Green and M. Gutperle, "Comments on three-branes", hep-th/9602077

[26] E. Verlinde, "Global aspects of electric-magnetic duality", Nucl. Phys. B455 (1995) 211, hep-th/9506011

[27] I. R. Klebanov and L. Thorlacius, "The size of $p$-branes", hep-th/9510200; S. S. Gubser, A. Hashimoto, I. R. Klebanov and J. M. Maldacena, "Gravitational lensing by $p$-branes", hep-th/9601057

[28] E. Witten, "Some comments on string dynamics", to appear in the proceedings of Strings '95, hep-th/9507121

[29] M. B. Green, "Pointlike states for type IIB superstrings", Phys. Lett. 329B (1994) 435, hep-th/9403040; T. Banks and L. Susskind, "Brane-antibrane forces", hep-th/9511194

[30] J. Polchinski and E. Witten, "Evidence for heterotic-type I string duality", Nucl. Phys. B460 (1996) 525, hep-th/9510169

[31] C. Vafa, "Evidence for F-theory", hep-th/9602022; D. R. Morrison and C. Vafa, "Compactifications of F-theory on Calabi-Yau threefolds - I", hep-th/9602114 